Instructions for authors, subscriptions and further details:

\title{
Apuntes sobre el Informe Coleman. Sobre la Difícil Convivencia de los Principios Igualitarios en un Mundo Desigual
}

Ildefonso Marqués Perales ${ }^{1}$

1) Universidad de Sevilla, Spain

Date of publication: June $25^{\text {th }}, 2016$

Edition period: June 2016-October 2016

To cite this article: Marqués Perales, I. (2016). Apuntes sobre el Informe Coleman. Sobre la Difícil Convivencia de los Principios Igualitarios en un Mundo Desigual. International Journal of Sociology of Education, 5(2), 107126. doi: 10.17583/rise.2016.2092

To link this article: http://dx.doi.org/10.17583/rise.2016.2092

\section{PLEASE SCROLL DOWN FOR ARTICLE}

The terms and conditions of use are related to the Open Journal System and to Creative Commons Attribution License (CC-BY) 



\section{Notes on Coleman Report. About the Difficult Coexistence of Equality Principles in a Non Equal World}

Ildefonso Marqués Perales

Universidad de Sevilla

(Received: 11 May 2016; Accepted: 28 May 2016; Published: 25 June 2016)

\section{Abstract}

Fifty years ago, "Equality of Educational Opportunity" was released in USA (Coleman et al., 1966). It is not an exaggeration to state that no educational report has had so far a strong impact in the national debate about social justice. Firstly, we are going to give an account about the main strengths of this report. To do that, we outline the historical scenario in which the report was born. Secondly, given that the main finding consisted of showing the importance of families in the educational process, we analyze the relationship the social class and educational attainment from the fifties to the first decade of this century in Spain. Moreover, we add a proxy variable of educational performance. Lastly, we draw the most relevant conclusions of such report in terms social philosophy and policy.

Keywords: Coleman Report, Schools, teachers, Families, Social Classes, Educational Attainment and Performance 


\section{Apuntes sobre el Informe Coleman. Sobre la Difícil} Convivencia de los Principios Igualitarios en un Mundo Desigual

Ildefonso Marqués Perales

Universidad de Sevilla

(Recibido: 11 de Mayo 2016; Aceptado: 28 de Mayo 2016; Publicado: 25 Junio 2016)

\section{Resumen}

Hace cincuenta años, se publicó en los EEUU el informe "Equality of Educational Opportunity" (Coleman et al., 1966). Sin ser exagerados, se puede señalar que ningún informe educativo ha tenido, hasta la fecha, un impacto similar en el debate sobre la justicia social de un país. En este artículo, vamos, por un lado, a dar cuenta de las principales virtudes de este informe. Para ello, esbozamos el escenario histórico que dio pie a su realización. En segundo lugar, dado que el principal hallazgo del Informe Coleman consistió en mostrar la importancia que tienen las familias en el proceso educativo, analizamos la relación que ha guardado la clase social y el logro escolar desde los años cincuenta del pasado siglo hasta la primera década del presente siglo. También, lo hacemos con una variable proxy del rendimiento educativo aunque en una franja de tiempo menos completa que la anterior. Por último, extraemos las conclusiones que, a nuestro juicio, cabe sacar del informe en materia de política y filosofía social.

Palabras clave: Informe Coleman, Centros escolares, profesorado, familia, clase social, logro y rendimiento escolar. 



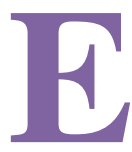

n la actualidad, el rol que cumple la educación como determinante principal de la posición social forma parte nuestro sentido común sociológico. Distintas investigaciones han demostrado que el impacto del origen del padre sobre el logro educativo es casi universal (Treiman y Yip, 1989) y que, a su vez, la educación es el principal canal para alcanzar una buena posición social (Breen, 2004). Esta relación que hoy vemos como evidente no siempre lo fue en el pasado. Dicho de otra forma, el hecho de que la escuela produjera sistemáticamente diferentes resultados según el origen social no fue fuente de controversia hasta una época muy reciente. Las controversias se hallaban en el reconocimiento por parte de las autoridades públicas de un desigual acceso a la enseñanza primaria y, sobre todo, a secundaria por parte de las poblaciones más pobres del país. En el caso de EEUU, la población rural y negra. Durante una buena época, la cuestión social giró en torno a la necesidad de una reforma educativa que garantizara la universalidad ante la enseñanza de forma gratuita y pública. Por poner un solo apunte: hay que tener en cuenta que hasta 1944, con la Education Act, no se habían abierto las aulas de forma gratuita a los estudiantes británicos que querían cursar secundaria.

Tras la Segunda Guerra Mundial, el desarrollo de un capitalismo más globalizado contribuirá a cambiar el modo en que las cuestiones educativas se iban solventando. Los procesos tecnológicos y de gestión adquieren una complejidad desconocida anteriormente. A lo largo de la primera mitad del siglo XX, los países occidentales van a decidir universalizar la educación más allá de los estrechos confines de la lectura y el cálculo básicos. Es, entonces, cuando se introducen las reformas comprehensivas. Como se sabe, su objetivo básico consistía en hacer el sistema educativo más equitativo, prolongando los programas comunes (hasta los dieciséis años en la mayoría de los casos). La razón era que la bifurcación temprana (tracking) de alumnos se consideraba como una fuente de desigualdad, que convertía al sistema escolar en un mecanismo de reproducción social, contraviniendo así su función de igualación de oportunidades o de mecanismo de promoción social.

En los EE.UU., tras la victoria demócrata en 1964, con la Higher Education Act (1965) se produce un vuelco en la financiación de la educación secundaria (Loss, 2012). Se intentará nivelar socialmente a los 


\section{Marqués - Apuntes sobre el Informe Coleman}

estudiantes con un sistema de beca de intereses bajos. Con estas reformas se intentaba remediar los males del sistema educativo tradicional reformándolo desde dentro. Para ello, se desplegaba un verdadero arsenal de tecnología pedagógica: diseños curriculares abiertos, trabajo en equipo y aprendizaje por auto-descubrimiento. Para dar cuenta de la fuerza de semejante cambio podemos acudir a la pirámide educativa del país. En 1950, un 2,6\% de la población no estaba escolarizada y un $45 \%$ había cursado estudios de primaria (terminándolos sólo un 20\%). Quince años después, un año antes del Informe Coleman, solo el $26 \%$ poseía un título de primaria. Las personas que habían cursado secundaria habían pasado del 38\% al 53\% (Barro y Lee, 2010). A este fenómeno lo denominamos expansión educativa y se reproducirá en la mayor parte de los países desarrollados del planeta.

\section{Los Primeros Estudios}

A pesar de la confianza inicial en la reforma comprehensiva de los sistemas educativos, los síntomas de la crisis de la educación no se hicieron esperar. En 1967 ya existía una clara conciencia de que los sistemas educativos necesitaban nuevas reformas. Ese mismo año se celebró en Virginia (EE.UU.) la Conferencia Internacional sobre la Crisis Mundial de la Educación. La cuestión ya iba estando más o menos clara: existían diferenciales en el acceso a la educación, principalmente, a etapa la secundaria.

En Francia, diferentes sociólogos van también poco a poco descubriendo esta relación y adentrándose en los mecanismos que la provocan. En el año 1962, Alain Girard y Roger Bastide hicieron una encuesta en la que presentaron las diferencias de acceso a la educación en base al origen social. Fue a partir de estos trabajos cuando Bourdieu y Passeron aprendieron que las desigualdades educativas no se explicaban por el nivel de sueldo, sino por el de instrucción. Pero no sólo eso. En 1964, el sociólogo francés Paul Clerc comprueba que no había ninguna asociación entre la frecuencia de la intervención de los padres con sus hijos y el nivel de éxito escolar Eran más bien la forma y la intensidad de la intervención las que contaban a la hora de obtener un mejor resultado escolar. En Les Héritiers (1964), Bourdieu y Passeron enriquecen con nuevas fuentes esta evidencia sociológica.

Poco a poco se fueron acumulando experiencias que indicaban que los 
nuevos métodos en los que tantas esperanzas se habían puesto en el pasado no estaban cambiando de forma radical la realidad educativa. Lo que preocupaba a los pioneros de la sociología de la educación puede resumirse de la siguiente forma: las sociedades capitalistas industrializadas tratan de acomodar dos principios de difícil reconciliación: el respeto a un orden de libertad y, a la vez, a un orden de igualdad. Bajo el primero de ellos, la familia es responsable de los hijos y, al igual que les puede transmitir las propiedades que disfrutan, pueden donarles pasivamente y activamente los recursos culturales que poseen. También, sus recursos sociales. Bajo el segundo, las sociedades deben proporcionar a los miembros que la forman unas condiciones sociales preliminares que les permitan acceder, si demuestran talento y se esfuerzan considerablemente, a los destinos sociales más deseados. La preocupación, por lo tanto, consiste en acoplar estos dos niveles. Como hemos señalado más arriba, la cuestión radicaba cómo asegurar la igualdad de oportunidades en una sociedad desigual? La respuesta parecía hallarse en el aumento de la inversión educativa. Si los alumnos de padres desventajados socialmente disponen de buenas escuelas para llevar a sus hijos, estos tendrían la posibilidad de medrar socialmente. Los diferenciales de acceso a la educación en sus etapas medias y superiores por clase social eran el producto de las condiciones materiales disímiles de las escuelas en las que se formaban a los alumnos. Era una cuestión de imputs escolares, de contrabalancear la báscula de las inversiones educativas.

\section{El Informe Coleman: Un Sistema Educativo Incrustado}

Es aquí donde cabe ubicar Equality of Opportunity (Coleman et al., 1966). Como es bien sabido, el popularizado como Coleman Report fue encomendado a una serie de investigadores asociados a la John Hopkins University, entre los que se encontraba James Coleman. Este fue financiado por el Comisionado de Educación de los EEUU en el contexto de implantación de la Ley por los Derechos Civiles de 1964. Bajo el mandato del demócrata Lydon B. Jonhson, la lucha contra la injusticia racial y la pobreza quedó enmarcada en el programa Great Society. Con éste, se intentaba dar un paso más, tratando de erradicar las injusticias que por razón de origen étnico y racial se producían aún en los EEUU. Una década antes, 


\section{Marqués - Apuntes sobre el Informe Coleman}

la Corte Suprema del país, en el Caso Brown contra el Consejo de Educación de Topeka (1954), había declarado que la separación de las escuelas entre blancos y negros atentaba contra la igualdad de oportunidades.

El Informe Coleman fue concebido, entonces, como una prueba de evaluación del sistema escolar norteamericano en términos de justicia social. Nunca anteriormente se había realizado semejante estudio, ni en los EEUU ni en ningún otro lado. Las cifras de la muestra todavía sorprenden: 600.000 estudiantes y 3.100 escuelas analizadas. Profesores, directores y superintendentes fueron entrevistados. Dos advertencias cabría hacer antes de comentar más profundamente el informe. En primer lugar, el intervalo entre la recogida de información y su posterior análisis fue mínimo. En segundo lugar, el análisis no tuvo una finalidad científica. Fue realizado para burócratas.

\section{Importan las Familias y los Ambientes que las Rodean}

Los resultados obtenidos por el informe fueron descorazonadores tanto para los científicos de la educación como para los responsables políticos. Evidenciaban que el desempeño escolar es refractario en gran medida a los diferentes inputs que a la sazón especialistas de la educación recomendaban en su recetario reformista. La novedosa y celebrada filosofía pedagógica no ayudaba a que los estudiantes obtuvieran mejores resultados. Los buenos resultados escolares se alcanzaban cuando los padres de los alumnos eran profesionales y directivos (White-Collars, en el lenguaje de la época) y no cuando eran obreros, independientemente de si sus escuelas estaban más y mejor dotadas.

"Las medidas usuales de calidad educativas consideradas como recursos para el aprendizaje (gasto por alumno, educación para el profesorado, tamaño de la biblioteca del colegio) mostraban poca relación con el logro escolar cuando los estudiantes de bagaje similar eran comparados, mientras que las diferencias entre los distintos bagajes familiares mostraban una relación importante con el rendimiento educativo" (Coleman, 1996, p. 19).

Dicho de otra forma, comparado con el impacto que tenía el origen social 
en el rendimiento escolar, la influencia de gasto por alumno, los gabinetes pedagógicos, el tamaño de la escuela, los volúmenes por librería, las características de los maestros o la existencia de la elección temprana, era muy reducida. El principal predictor del rendimiento escolar era la familia y los recursos de los que disponía.

Es cierto que el trabajo adolece de preocupación teórica. No en vano era un informe realizado, como hemos dicho, por el Comisionado de Educación de los EE.UU. Hay que tener en cuenta que lo interesante, en términos sociales, es la relación que guarda el rendimiento con el logro escolar. La razón era bien sencilla. El escaso rendimiento conduce a un escaso logro y este a un bajo logro ocupacional. De ahí que la evaluación del sistema escolar pudiera considerarse un examen a la igualdad de oportunidades en los EEUU. Hemos de esperar algunos años aún para que la relación entre origen social y logro escolar se asiente empíricamente y surjan teorías capaces de dar cuenta de los mecanismos implicados.

El informe nos venía a decir que la vida escolar de un país se inserta en su estructura social. No constituía una esfera independiente. Las divisiones raciales, económicas y sociales que fracturaban los EEUU se dejaban sentir "golpeando" los pupitres de sus aulas. Las escuelas norteamericanas estaban socialmente y racialmente tan segregadas como lo estaba su sociedad. Los alumnos blancos obtenían mejores resultados que aquellos que pertenecían a las minorías raciales. Dicho en términos empleados por la sociología económica (Polanyi, 1997), el sistema escolar norteamericano se hallaba incrustado en la sociedad americana e interrelacionado en sus instituciones. Esta fue la principal conclusión que se extrajo del Informe Coleman y, puede decirse, sin ir demasiado lejos, que su legado aún permanece intacto.

\section{¿Importan los Centros?}

Dado que la varianza dentro de las escuelas era muy superior a la varianza entre ellas, se deducía de todo ello que los centros importaban poco. Dicho de otro modo, una vez controlado estadísticamente el efecto del origen social, el impacto de las escuelas era muy reducido. Esta relación socavaba las bases de la acción pedagógica. Pese a que no se decía, todo parecía indicar que la posibilidad de transformación de la realidad social por parte de las reformas educativas era muy limitada. Profesores, pedagogos y políticos 


\section{Marqués - Apuntes sobre el Informe Coleman}

veían así su radio de acción disminuido. Existen puntos a favor y en contra de este resultado. En contra puede señalarse que existen medidas escolares como la repetición de curso y la selección temprana (tracking) que tienen un impacto negativo en la igualdad escolar. No obstante, los estudios sobre movilidad social confirman que, en muchos países, el alcance de las reformas comprehensivas es limitado debido a la persistencia que presentan las desigualdades educativas (Goldthorpe, 2012). En segundo lugar, se daba a entender que la escuela recompensaba a aquellos que gozaban de un ambiente social rico y castigaba a aquellos que provenían de las capas sociales menos privilegiadas. Esto alimentó empíricamente a las teorías de la reproducción y la correspondencia.

La literatura sobre el papel que cumplen los centros en la vida escolar es ingente, sin embargo, los resultados obtenidos por Coleman han sido verificados en las últimas cinco décadas (Plowden, 1967; Hanushek, 2010). Los datos del informe PISA han arrojado mucha luz sobre esta relación. En las pruebas de comprensión matemática, la cantidad de varianza explicada por los centros (teniendo en cuenta el origen social de los estudiantes) varía según los países considerablemente dada la gran variedad en sus instituciones sociales. De media en la OCDE, la varianza intra-escuelas es aproximadamente de un $63 \%$ y las inter-escuela 37\%. En nuestro país, la diferencia es menor (en torno a un 17\%) que los demás países de la OCDE (PISA, 2012). No obstante, si descontamos por completo el origen social de los alumnos, la varianza es aún menor. Aun así sí es cierto existen indicadores específicos que han demostrado ser significativos, lo que no invalida que los resultados obtenidos en el informe Coleman se mantienen muy vivos (Hanushek, 2010).

\section{¿Importan los Profesores?}

En lo que concierne a los profesores, las investigaciones emprendidas hasta la fecha no han conseguido averiguar cuáles son las características que debe tener un buen profesor. "La credencial de los profesores, el hecho de haber obtenido un certificado de estudios avanzados, si fueron a un college o a una universidad, si se recibe más o menos tutoría o desarrollo personalizado, no guardan casi ninguna relación con la efectividad de los profesores en la clase" (Hanushek, 2010, p. 24) ¿Significa esto que los profesores no 
cuentan? Sería arriesgado pensar así. Todos hemos asistido a clase y hemos comprobado in situ que la misma materia puede ser aprendida fácilmente o ser un verdadero tedio según sea el profesor que la imparta. Además, existe un problema importante: los estudiantes que reciben las clases son muy diferentes en términos sociológicos, lo que hace, sin duda, muy complicado su examen. Es probable que los instrumentos de medición fallen y estemos prestando atención a las variables erróneas. No obstante, tras Coleman, hoy en día sabemos que los recursos no son importantes a no ser que sean bien empleados por los profesores. Las investigaciones contemporáneas han puesto, por ello, especial énfasis en el rol que pueden desempeñar los incentivos (Hanushek, 1994; Heckman \& Neal, 1996).

\section{Tres Críticas Pertinentes y Tres Justificaciones Insuficientes}

Pese a que aún puede insistirse en la validez del Informe Coleman, ello no obsta para que puedan realizarse algunas críticas pertinentes. Nosotros enumeramos tres de distinta naturaleza:

1) El movimiento de escuelas eficaces ha demostrado que algunas variables relacionadas con los centros son muy pertinentes a la hora de disminuir el efecto del origen social sobre el destino educativo de los alumnos. En efecto, desde la pronta respuesta de Weber (1971), esta corriente ha adquirido mayor fuerza, sin embargo, existe una clara dificultad para estandarizar las variables que emplean. Además, las experiencias exitosas se han limitado a espacios concretos que no dejan rastro objetivo en el agregado de escuelas.

2) En ciencias sociales, no existe relación empírica que no está sustentada bajo un mecanismo que la explique. Es pertinente, entonces, las objeciones realizadas sobre la "caja negra" inserta bajo el modelo input-output. Aquí, puede señalarse que el informe fue realizado con premura y en aras de presentar un informe empíricamente neutral.

3) Julio Carabaña atribuye una carencia a los estudios que, como el de Coleman (2015), prescinden del aprendizaje para centrarse en las aptitudes. Es lo que denomina la "paradoja de Jencks". Las pruebas miden las destrezas y no los contenidos. Sin dudarlo un segundo, es una crítica muy pertinente y acertada. No obstante, se podría señalar que estandarizar pruebas basadas en aptitudes es mucho más sencillo (y conveniente en 


\section{Marqués - Apuntes sobre el Informe Coleman}

términos políticos) que en contenidos.

\section{La Persistencia de las Fuerzas Sociales}

Como hemos dicho en nuestra introducción, como tributo a Coleman, vamos a analizar la influencia que ha tenido el origen social en el logro y el rendimiento educativo en el caso de España a lo largo de seis décadas. Para mostrar la estabilidad de la desigualdad educativa a través del tiempo vamos a analizar su evolución desde los años cincuenta del pasado siglo hasta la primera década del presente siglo. Contemplamos, por consiguiente, incluso un periodo anterior a la expansión educativa.

La teoría liberal vaticina que, puesto que la modernización entraña una demanda creciente de trabajadores cualificados gracias al desarrollo tecnológico (Acemoglu, 2002), la relación entre origen social y logro social se está debilitando muy rápidamente. La teoría de la reproducción, por el contrario, predeciría una relación persistente (Bourdieu y Passeron, 1970).

Codificamos las clases sociales como viene siendo usual, según el esquema EGP que mostramos en el Apéndice. Esta será nuestra variable independiente. El nivel educativo, que tomamos como variable dependiente, está formado por cuatro categorías agrupadas del ISCED: 0-1 (Primaria o menos), ISCED 2ab (secundaria básica), ISCED 2c (secundaria postobligatoria) y 3 (universitaria). Formamos cuatro cohortes de los nacidos entre: 1914-31, 1932-1946, 1947-61 1962-1976. Dado que hemos seleccionado a la población activa de 35 a 70 años, esto supone analizar a las personas que accedieron al mercado laboral desde principios de los cincuenta hasta la primera década del presente siglo. A partir de 35 años (edad de madurez laboral), son pocas las personas que estudian.

Sumamos las muestras compuestas por cuatro de las encuestas con más unidades que se han realizado en España: la encuesta de Evaluación de las necesidades sociales (Estudio 1737, 1988), la Encuesta Socio-demográfica (1992) y la Encuesta de Condiciones de Vida (2005 y 2012). En total, el análisis se ha realizado sobre cerca de 80.000 personas.

Creamos tres modelos. En el primero de ellos, modelamos una ecuación en la que no existiría cambio temporal en el efecto del origen social sobre el logro educativo. En el segundo, permitimos un cambio uniforme por cohorte. En el tercero, añadimos una restricción lineal. Todos estos modelos 
que hemos diseñado son los modelos usuales log-linear empleados en la literatura científica.

La tabla 1 muestra los resultados obtenidos tanto para la población masculina como para la femenina. Como se aprecia, el modelo de constancia no es mejor que el resto de los modelos que implican un cambio, ni para los hombres ni para las mujeres. Las diferencias entre los dos modelos que imprimen, como hemos dicho, un cambio de naturaleza uniforme $y$ uniforme-lineal ajustan mejor.

Tabla 1

El impacto del origen social sobre el logro escolar. Ajuste de los modelos de Constancia, Cambio Uniforme, Cambio Uniforme Lineal

\begin{tabular}{llllll}
\hline Hombres & $\mathbf{L}_{\mathbf{2}}$ & GL & $\mathbf{p}$ & DI & BIC \\
\hline $\begin{array}{l}\text { Constancia } \\
\text { Cambio }\end{array}$ & 254,15 & 54 & 0 & 2,06 & $-327,23$ \\
$\begin{array}{l}\text { Uniforme } \\
\text { Restricción }\end{array}$ & 229,77 & 51 & 0 & 1,96 & $-319,31$ \\
linear & 231,3 & 53 & 0 & 1,95 & $-339,31$ \\
\hline Mujeres & & & & & \\
\hline $\begin{array}{l}\text { Constancia } \\
\text { Cambio }\end{array}$ & 231,48 & 54 & 0 & $2,01 \%$ & $-329,66$ \\
$\begin{array}{l}\text { Uniforme } \\
\text { Restricción } \\
\text { linear }\end{array}$ & 206,99 & 51 & 0 & $1,92 \%$ & $-322,98$ \\
\hline
\end{tabular}

Fuentes: Encuesta de Evaluación de las necesidades sociales (1988), Encuesta Socio-demográfica (1991) y Encuesta de Condiciones de Vida (2005 y 2012).

Esto indica que ha existido una trasformación en el efecto del origen sobre el destino por cohorte. No obstante, ya que la constricción lineal apenas aporta una mejora, mostramos la evolución de los parámetros logmultiplicativos (Xie, 1992). Nuestro modelo fija la primera cohorte con una fuerza de asociación de uno. Superar este número supone un fortalecimiento de la relación y una disminución equivale a un debilitamiento. Dado que ninguno de los parámetros supera al parámetro de la primera cohorte, 


\section{Marqués - Apuntes sobre el Informe Coleman}

podemos interpretar en términos porcentuales la disminución de éstos por cohorte.

En consecuencia, son las cohortes nacidas en los años sesenta y setenta las más perjudicadas pues la asociación deja de mejorar. Hay que tener en cuenta que la Ley General de Educación fue implantada en los años setenta $\mathrm{y}$, de acuerdo con alguna investigación precedente, ésta pudo haber tenido un efecto adverso en la igualdad (Carabaña, 2004). Cuando uno presta una atención al comportamiento por clase social, observa que las clases sociales manuales de la última cohorte alcanzaron el ISCED 2ab en mayor proporción que la clase de servicio, administrativa y la pequeña burguesía. Estos datos están en consonancia con el MMI hipótesis (desigualdad mantenido al máximo) (Raftery \& Hout, 1993) ya que una vez que la igualdad en los niveles obligatorios de la educación se produce cuando las clases altas han saturado este nivel.

Como se aprecia, a medida que las cohortes avanzan, existe una mejora de la fluidez educativa. Tanto en el caso de los hombres como de las mujeres, desde la primera a la última cohorte, la mejora fue de un $18 \%$ aproximadamente. En los hombres, el cambio es más significativo en aquellos que accedieron al mercado en la década de los sesenta y setenta. En el caso de las mujeres, también podemos incluir la década de los ochenta. En seis décadas, la asociación se ha debilitado alrededor de $6 \%$ por cohorte en los hombres y un $7 \%$ por cohorte en las mujeres. Sin embargo, el cambio apenas en apreciable en las dos últimas cohortes.

Estos datos excluyen cualquier interpretación contundente de la evolución histórica de la evolución de la desigualdad en España. No existe una mejora ni un empeoramiento drástico. Sólo existe una ligera reducción de la desigualdad escolar de los cincuenta hasta la primera década del presente siglo. 


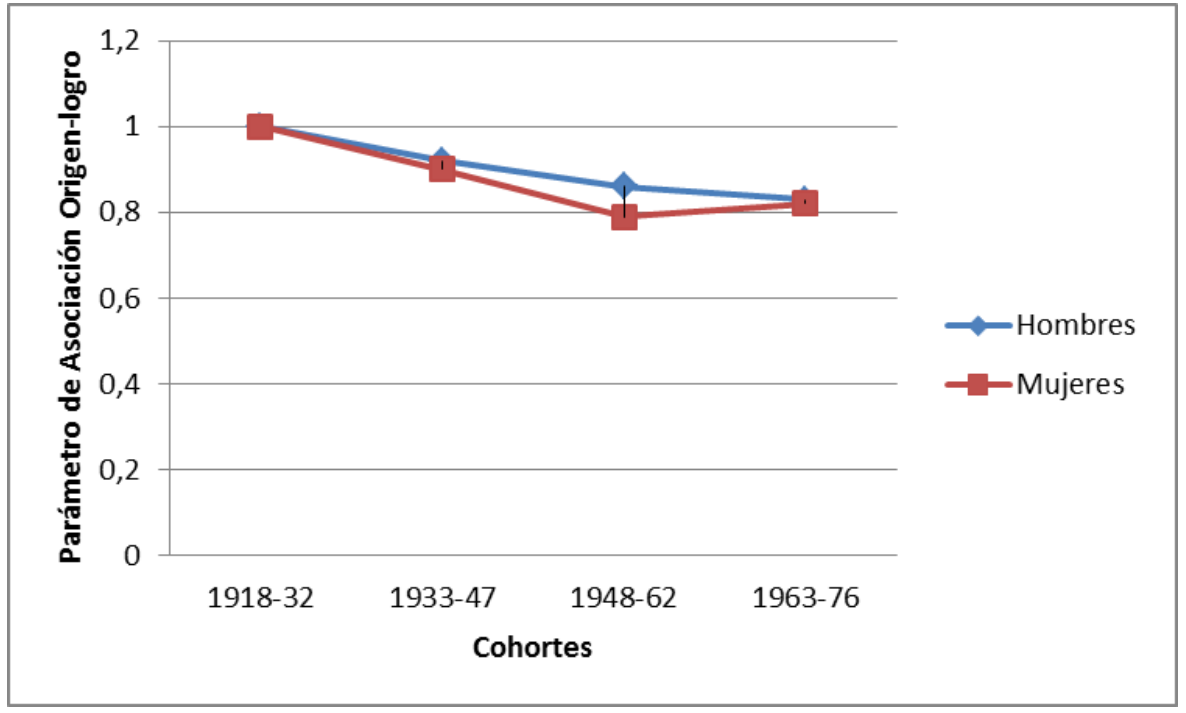

Figura 1

Efecto del origen sobre el logro educativo

Fuentes: Encuesta de Evaluación de las necesidades sociales (1988), Encuesta Socio-demográfica (1991) y Encuesta de Condiciones de Vida (2005 y 2012).

Tabla 2

El impacto del origen social sobre el rendimiento escolar. Modelos de Constancia y Cambio Uniforme

\begin{tabular}{lllcll}
\hline & $\mathbf{L}$ & $\mathbf{G L}$ & $\mathbf{p}$ & $\mathbf{B I C}$ & DI \\
\hline Constante & 121,59 & 96 & 0,03993597 & $-712,25$ & $\mathbf{4 , 5 3}$ \\
$\begin{array}{l}\text { Cambio } \\
\text { Uniforme }\end{array}$ & 120,92 & 92 & 0,02327711 & $-678,18$ & $\mathbf{4 , 5 9}$ \\
\hline
\end{tabular}

Fuente: Encuesta de clases sociales y estructura social (2006) 
Hemos mostrado la relación que guarda el origen social con el logro escolar. No obstante, Coleman en su célebre informe evaluó no el logro, sino el rendimiento de los alumnos en pruebas estandarizadas. En España, existen pruebas de rendimiento tanto para alumnos de primaria como de secundaria, tanto nacionales como internacionales. No obstante, estas pruebas son recientes. Nuestro objetivo sería comparar el impacto del origen social en el rendimiento a lo largo de un mayor periodo de tiempo. La Encuesta de Clases Social y Estructura Social (2006) posee una pregunta que nos puede servir de variable proxy del rendimiento educativo. La pregunta es la siguiente:

¿Cómo eran las notas que sacaba usted en el colegio-instituto-escuela donde estudiaba? (cuando tenía usted 16 años o antes)

- No tiene estudios

- Sacaba muy buenas notas

- Buenas

- Regulares

- Malas

- Sacaba muy malas notas

Como podemos ver en la figura 2, la relación entre origen social y rendimiento escolar presenta aún más estabilidad que con aquella del logro escolar. Los parámetros apenas fluctúan y, lo que es más importante, no alcanzan ninguna dirección. De hecho, la tabla 2 nos señala que las diferencias entre el modelo uniforme y el constante no son significativas. Dicho de otra forma, el impacto de las clases sociales en el rendimiento educativo es constante a través de las cohortes estudiadas. 


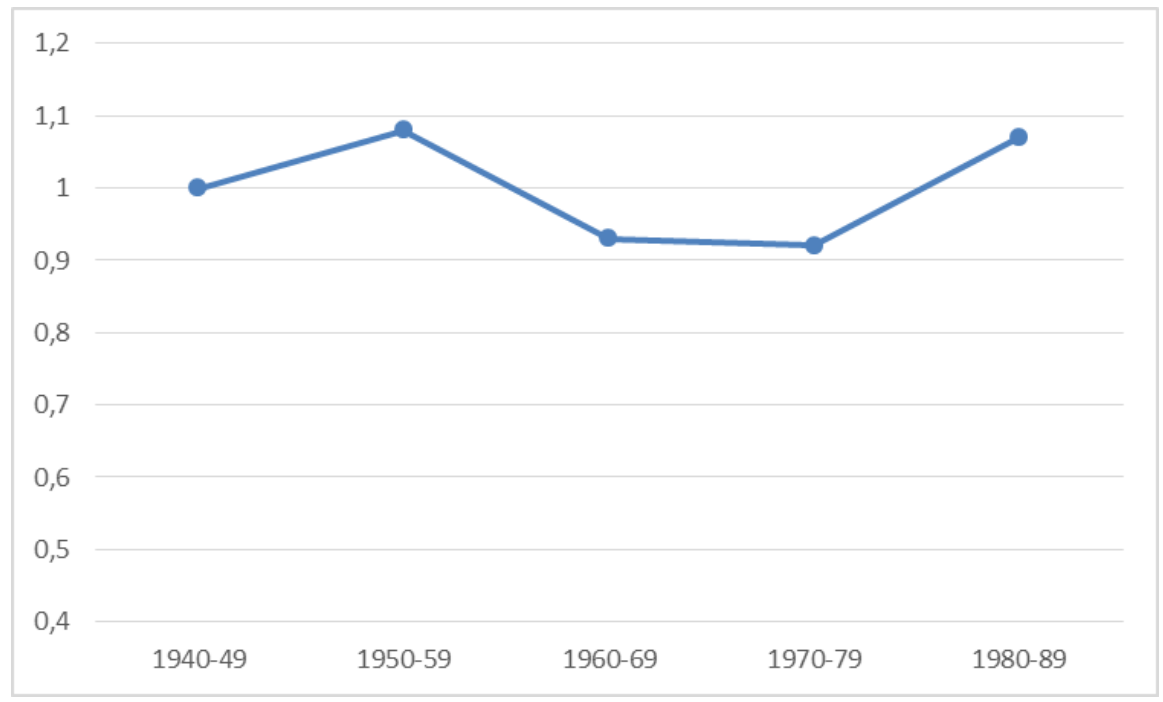

\section{Figura 2}

Efecto del origen sobre el rendimiento educativo

Fuente: Encuesta de clases sociales y estructura social (2006)

\section{Conclusiones}

En este artículo, hemos tratado de extraer las lecciones fundamentales del Informe Coleman. Hemos subrayado que el énfasis puesto en las familias a la hora de explicar el rendimiento escolar había constituido un importante giro heurístico. Hemos revisado la relación que ha guardado la clase social y el logro escolar desde los años cincuenta del pasado siglo hasta la primera década del presente siglo. También, lo hemos hecho con una variable proxy del rendimiento escolar.

Una de las grandes cuestiones que cabía preguntarse tras la publicación del Informe Coleman radicaba en si era posible garantizar, respetando un orden de libertad, la igualdad de oportunidades. Tan solo nueve años después de su publicación, los investigadores Featherman, Jones \& Hauser (1975) descubrieron que las tasas de movilidad absoluta en un país diferían unas de otras, pero no se distanciaban en cuanto a las tasas de movilidad de 


\section{Marqués - Apuntes sobre el Informe Coleman}

circulación (o relativas). Las diferencias en las tasas de movilidad absoluta se debían a variables (demográficas, tecnológicas y económicas) que poco tienen que ver con los procesos de transmisión de privilegios de clase a través de generaciones (Marshall, Swift y Roberts, 1997, p. 236). "En sociedades industriales - señalan estos autores - con economía de mercado y sistema de familia nuclear (las tasas de movilidad relativa) son básicamente las mismas" (Featherman et al., 1975, p. 340).

Como se sabe, en las sociedades contemporáneas, las oportunidades de los individuos vienen canalizadas a través de dos vías: aquella que es el producto de su origen social y aquella que es el resultado de la expresión de su esfuerzo y talento. La sociedad asigna a la escuela la potestad de decirnos quién posee estas cualidades. Para ello, ha de nivelar las condiciones de partida de los individuos. Sin embargo, esto no es fácil, ya que para garantizar la igualdad de oportunidades la intervención social habría de introducirse en la familia. Semejantes reformas sociales son consideradas por una buena parte de la población como radicales por lo que es harto probable que no fueran bien recogidas. El problema, por tanto, reside en el simple hecho de que origen social y educación se hallan íntimamente ligados. Las oportunidades no sólo son el reflejo de la transmisión de la propiedad -que sería normal una economía capitalista- en un orden de libertad, sino también de un acceso desigual a la cima de jerarquía escolar.

Las reformas escolares casi siempre fracasan en su intento de transformación de una realidad que las desborda. Solo aquellos países que históricamente se han embarcado en una reforma profunda y radical de sus instituciones han conseguido nivelar la relación entre el origen y el logro escolar (Erikson \& Goldthorpe, 1992). Más de treinta años les costó a los socialistas suecos invertir esta relación.

Por otro lado, hay que señalar que, aun imaginando que la influencia de origen social en el logro escolar se evaporara, los grupos sociales con mayores ventajas encuentran otras vías para asegurarse trasmitir sus recursos a sus hijos. Boudon ya nos advirtió que una menor desigualdad educativa no tenía que traducirse obligatoriamente en una menor desigualdad económica (Boudon, 1983). España es un país cuya demanda de puestos cualificados es menor a la oferta universitaria. Esto hace que tengamos la tasa de sobreeducación mayor de Europa. En estas condiciones, a igual título universitario, el origen social presenta una ventaja (codificado a través del 
capital cultural y social) (Marqués \& Gil, 2015). Además, y esto no hay que olvidarlo, la actividad económica de nuestro país está dominada por la pequeña empresa. Muchos pequeños empresarios acceden a éstas bien a través de la aportación económica de algunos de sus familiares, o bien por medio de la cesión directa del negocio. En ninguno de los dos casos la educación es necesaria.

Existen medidas de carácter social que serían enormemente beneficiosas para la vida escolar. Hoy en día, sabemos que las inversiones escolares dependen la capacidad de los individuos y sus familias para hacer cálculos a largo plazo. Una familia en la que el trabajo aparece y desaparece origina aversión al riesgo en sus apuestas educativas. Los altos costes de oportunidad exigen que los primeros empleos no puedan ser rechazados por lo que se abandona la escuela prematuramente (Erikson \& Jonsson, 1996). La reducción del paro, el control de la precarización laboral y de la temporalidad tendrían un impacto muy positivo en la vida escolar. Mayor, sin duda, que la inversión en recursos como ya nos indicó Coleman.

\section{Referencias}

Acemoglu, Daron (2002). Technical Change, Inequality, and the Labor Market. Journal of Economic Literature, 40(1), 7-72.

Barro, R. Lee, J.W. (2010). Data Set, http://www.barrolee.com.

Boudon, Raymond (1983). La desigualdad de oportunidades: la movilidad social en las sociedades industriales. Barcelona: Laia.

Bourdieu P., Passeron J.-C. (1964). Les Héritiers. Les étudiants et la Culture. Paris: Minuit, coll. Le sens commun.

Bourdieu, P., \& Passeron, J. C. (1970). La reproduction: Éléments pour une théorie du système d'enseignement. Paris: Éditions de Minuit.

Breen, R. (2004). Social Mobility in Europe. Oxford: Oxford University Press.

Carabaña, J. (2004). Educación y movilidad social. Vicenç Navarro y Águeda Quiroga (eds.), El Estado de Bienestar en España, Madrid: Tecnos.

Carabaña, J. (2015). La inutilidad de Pisa para las escuelas. Madrid: Los Libros de la Catarata. 
Coleman, J.S.; Campbell, E. Q.; Hobson, C.J.; McPartland, J., Mood, A.

M., Weinfeld, F. D., York, R. (1966). Equality of educational

opportunity. Washington, DC: US Department of Health, Education

\& Welfare, Office of Education.

Clerc, P. (1964). La famille et l'orientation scolaire au niveau de la

sixième. Population, p. 627-672.

Erikson, R. \& Goldthorpe, J.H. (1992). The Constant Flux. Oxford:

Clarendon Press.

Erikson, R., \& Jonsson, J. O. (1996). Can education be equalized?: The

Swedish case in comparative perspective. Boulder, Colo: Westview

Press.

Featherman, D.L., Jones F.L, and Hauser, R.M. (1975). Assumptions of

Social Mobility Research in the US: The Case of Occupational

Status', Social Science Research, 4, 329-360.

Girard, A. y Bastide, H. (1963). La stratification sociale et la

démocratisation de l'enseignement, Population, 3, repris in : INED (1970), op. cit. (91-128).

Goldthorpe, J. (2012). Understanding - and Misunderstanding - Social Mobility in Britain: The Entry of the Economists, the Confusion of

Politicians and the Limits of Educational Policy, Oxford Institute of Social Policy and Nuffield College, University of Oxford.

Hanushek, E. (2010). Generalizations about Using Value-Added Measures of Teacher Quality. Eric A. Hanushek, Steven G. Rivkin. American Economic Review, 100(2), 267-271.

Hanushek, E. (2016). What Matters for Student Achievement, Education Next, Spring, 16(2).

Heckman, James, and Derek Neal. (1996). Coleman's Contributions to Education: Theory, Research Styles, and Empirical Research. In Jon Clark, ed., James S. Coleman. London: Falmer Press

Loss, C.P. (2012): Between Citizens and the State, Princeton: Princeton University Press.

Marshall, G., Swift, A. y Roberts, S. (1997). Against the odds? : social class and social justice in industrial societies. Oxford scholarship online. Oxford : Clarendon Press.

Marqués Perales, I., Gil-Hernández, C.J. (2015). Origen social y sobreeducación en los universitarios españoles: ¿es meritocrático el 
acceso a la clase de servicio? Revista Española de Investigaciones Sociológicas, 150, 89-112. doi:10.5477/cis/reis.150.89

Polanyi, K. (1997). La gran transformación, Madrid, La Piqueta.

Raftery, A.E., \& Hout, M. (1993). Maximally Maintained Inequality.

Sociology of Education, 66, 41-62.

Treiman, Donald J., and Kam-bor Yip. (1989). Educational and

Occupational Attainment in 21 Countries. Pp. 373-394 in Cross-

National Research in Sociology, edited by Melvin L. Kohn (ASA

Presidential Series). Beverly Hills: Sage Publications.

Weber, G. (1971). Inner-city children can be taught to read: four successful schools. Washington, DC: Council for Basic Education.

Xie, Y. (1992). The Log-Multiplicative Layer Effect Model for Comparing Mobility Tables. American Sociological Review, 57, 380-395.

Ildefonso Marqués Perales is Professor at Department of Sociology at University of Sevilla

Contact Address: Direct correspondence to Ildefonso Marqués Perales, Department of Sociology, Faculty of Education, University of Sevilla, C/ Pirotecnia, S/N, 41013, Sevilla, Spain. E-mail: 


\section{Apéndice}

\begin{tabular}{|c|c|c|}
\hline $\begin{array}{l}\text { Número } \\
\text { de las } \\
\text { clases }\end{array}$ & Nombre de las clases & Ocupaciones genéricas \\
\hline $\mathrm{I}+\mathrm{II}$ & Clase de servicio & $\begin{array}{l}\text { Directivos de grandes empresas y } \\
\text { profesionales liberales. }\end{array}$ \\
\hline IIIab & $\begin{array}{l}\text { Rutinaria no manual } \\
\text { (para simplificar: clase } \\
\text { administrativa) }\end{array}$ & $\begin{array}{l}\text { Administrativos, técnicos, empleados en trato } \\
\text { directo con el público y trabajadores en } \\
\text { servicios de rango alto así como empleados } \\
\text { en trato directo con el público de rango bajo y } \\
\text { trabajadores en servicios de rango bajo. }\end{array}$ \\
\hline IVab & Pequeña burguesía & Gerentes de empresas pequeñas, autónomos \\
\hline $\mathrm{IVb}$ & $\begin{array}{l}\text { Pequeña burguesía } \\
\text { agrícola }\end{array}$ & Pequeños propietarios agrícolas. \\
\hline $\mathrm{V}+\mathrm{VI}$ & $\begin{array}{l}\text { Supervisores y } \\
\text { trabajadores } \\
\text { cualificados. }\end{array}$ & $\begin{array}{l}\text { Trabajadores cualificados en la agricultura, la } \\
\text { pesca y ganadería, artesanos, trabajadores } \\
\text { cualificados en industrias manufactureras, de } \\
\text { la construcción, de la minería y operadores de } \\
\text { instalación y maquinaria. }\end{array}$ \\
\hline VIIa & $\begin{array}{l}\text { Trabajadores no } \\
\text { cualificados }\end{array}$ & $\begin{array}{l}\text { Empleados domésticos, personal de limpieza, } \\
\text { conserjes, limpiacristales, ordenanzas, peones } \\
\text { de la construcción, minería, de las industrias } \\
\text { manufactureras y de los transportes, } \\
\text { jornaleros. }\end{array}$ \\
\hline
\end{tabular}

VIIb Trabajadores agrícolas Jornaleros 